\title{
A Study to Evaluate the Vertical Dimension at Occlusion Using the Intercanthal Width as a Reference
}

\author{
Manoj Shetty ${ }^{1}$, Rishabh Gupte ${ }^{2}$, Nivya John ${ }^{3}$
}

\begin{abstract}
Aims and objectives: The study aimed to record the distance between the inner and outer canthus of the eye and to compare it with the distance between the base of the nose to the lower border of the lower lip which can later be used to measure vertical dimension in occlusion. Materials and methods: Two hundred dental students comprising males and females between 18 years and 25 years of age-group constituted the samples for this study. The intercanthal distance (distance between medial and lateral canthus of the eyes) and subnasale (base of the nose) to the lower border of lower lip distances were measured by the digital caliper when the patient closed in centric occlusion. In this manner, measurements were made in all the samples and data obtained were tabulated for further analysis.

Results: The coincidence of occlusal vertical dimension with intercanthal width among the male and female categories was 76 for males and 107 for females out of the total of 183. A 91.5\% accuracy was observed in both sexes which suggests that intercanthus distance can be used as a guide to developing active vertical dimension in edentulous subjects. This was done by adjusting the height of the occlusal rims and measuring the distance between the base of the nose to the inferior border of the lower lip till it equals the intercanthus distance. This method was later verified by clinical judgment.

Conclusion: Within the limitations of the study, it was concluded that the intercanthus distance can be used as a guide to developing active vertical dimension in edentulous subjects.

Clinical significance: The distance between the outer and inner canthus can be a reliable landmark, which can be used for anthropometric measurements to record vertical dimension in edentulous patients requiring complete dentures along with other existing methods.

Keywords: Intercanthus width, Jaw relation, Maxillomandibular relation prosthodontics, Vertical dimension at occlusion.

World Journal of Dentistry (2021): 10.5005/jp-journals-10015-1866
\end{abstract}

\section{INTRODUCTION}

Innumerable methods have been advocated to assist in recording vertical dimensions in complete denture patients and yet it appears that none of these methods are foolproof. ${ }^{1}$ Ultimately, clinical judgment decides the appropriate vertical dimension required for a particular subject.

Two methods that are commonly advocated are either based on the knowledge of physiologic rest position or the one which depends on the closest speaking space. Few other methods assist the dentist to arrive at a tentative vertical dimension of the jaws. ${ }^{2}$ These are pre-extraction records and methods based on craniofacial measurements. Pre-extraction record is not available for all subjects and one has to therefore depend on the facial proportions and the relationship existing between them. ${ }^{3}$

A brief review of the literature shows that almost all the facial landmarks have been utilized for anthropometric measurements (Fig. 1). ${ }^{4}$ Various measurements are as follows:

- The interpupillary distance.

- Outer canthus of one eye to inner canthus of the other eye.

- Distance from the brow to chin.

- Distance from the outer canthus to the angle of the mouth.

- Distance from the base of the nose to the lower border of the chin.

- Distance from the tip of the nose to chin.

- Distance from the external auditory meatus to the eye.

Measurements are also done by dividing the face into thirds. Some of these have been useful during the clinical recording of vertical dimension in denture patients. ${ }^{5}$
${ }^{1-3}$ Department of Prosthodontics and Crown and Bridge, AB Shetty Memorial Institute of Dental Sciences (NITTE Deemed to be University), Mangaluru, Karnataka, India

Corresponding Author: Nivya John, Department of Prosthodontics and Crown and Bridge, AB Shetty Memorial Institute of Dental Sciences (NITTE Deemed to be University), Mangaluru, Karnataka, India, Phone: +91 9742024358, e-mail: nivyajohn@nitte.edu.in

How to cite this article: Shetty M, Gupte R, John N. A Study to Evaluate the Vertical Dimension at Occlusion Using the Intercanthal Width as a Reference. World J Dent 2021;12(5):409-412.

Source of support: Nil

Conflict of interest: None

The outer canthus of the eye has often been used in prosthodontics as a landmark for the eye ear plane to orient the horizontal plane and axis orbital plane. ${ }^{6}$ The inner canthus of the eye is one of the landmarks for craniometric measurements which has so far not been utilized as extensively. ${ }^{7}$

While using craniometric measurements, for recording passive vertical dimensions at rest, a distance measuring from the reference point in the nose to a reference point in the chin is usually taken as a standard..$^{8}$ This method is popularly followed in practice as well in teaching dental students. It is difficult to pinpoint or locate the prominent part in the chin or to standardize this reference point.

Alternatively, a more stable reference landmark such as the lower border of the lower lip and the junction of the vermilion zone with the lower lip can be made use to record the nose chin distance. 


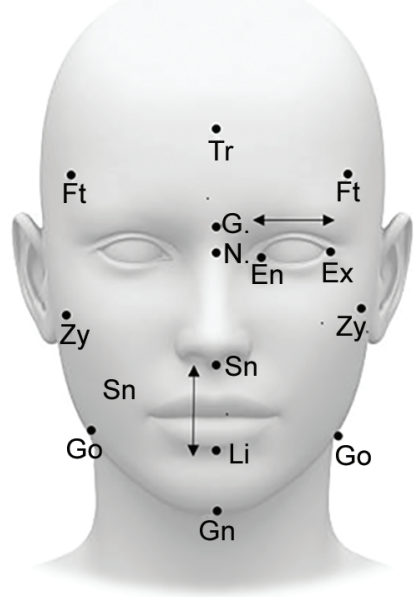

Fig. 1: Anthropological landmarks. Zy, zygoma; Tr, trichion, Ft, frontotemporal; G, glabella; N, nasion; En, endocath; Ex, hexocanth; Sn, subnasale; Go, gonion; Li, labiale inferius; Gn, gnathion

The measurement of intercanthus and outer canthus of the eye has been studied of its relationship with vertical dimension at rest in various studies. Misch in his anthropometric studies has stated that the anatomical landmark of the inner canthus is static and hence it is simpler to establish the intercanthal distance (ICD). Misch had studied the Caucasian population where they recommended using the length of twice the ICD to determine VDO. ${ }^{9}$ Similar studies have been done by Yaw et al. in Mongoloid race. ${ }^{10}$

Facial features can be influenced by race, ethnic background, nutrition, gender, age, etc. Sexual dimorphism is often noted in various facial features. A study done by Oladipo et al.'s study established the mean facial dimensions for ljaw children and adolescents were gender-dependent and sexually dimorphic. ${ }^{11}$

On casual observation, it is seen that the distance between the outer canthus and the inner canthus could be compared with the distance between the base of the nose (subnasale) to the lower border of the lower lip. If these two distances are the same then it could serve as one of the references for recording vertical dimensions.

In view of this, a study was undertaken to record the distance between the inner and outer canthus of the eye and to compare it with the distance between the base of the nose to the lower border of the lower lip among male and female Indian students.

\section{Materials and Methods}

Two hundred dental students comprising of males and females between 18 years and 25 years of age-group from Indian origin constituted the samples for this study. Informed consent was collected from the participants at the beginning of the study. A single investigator collected the measurements of all the participants.

The following criteria were used in the selection of samples:

- All the subjects had a definite centric stop with their natural dentition, to eliminate any change of vertical dimension.

- Patients who had undergone any major orthodontic treatment or suffered from a collapse of posterior bite due to loss of teeth were not included.

- Patients with severe class II, class III malocclusions were excluded.

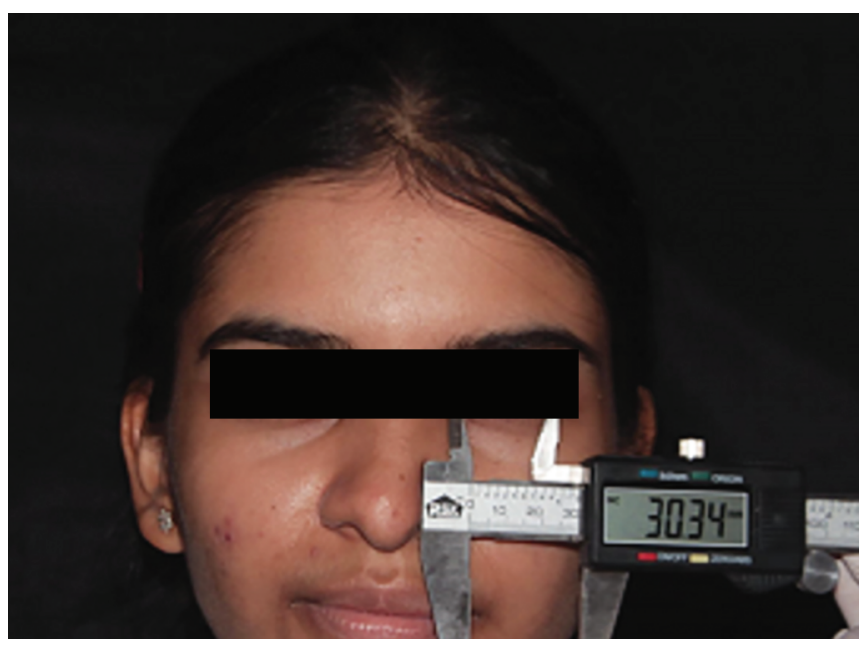

Fig. 2: Measurement of intercanthal distance

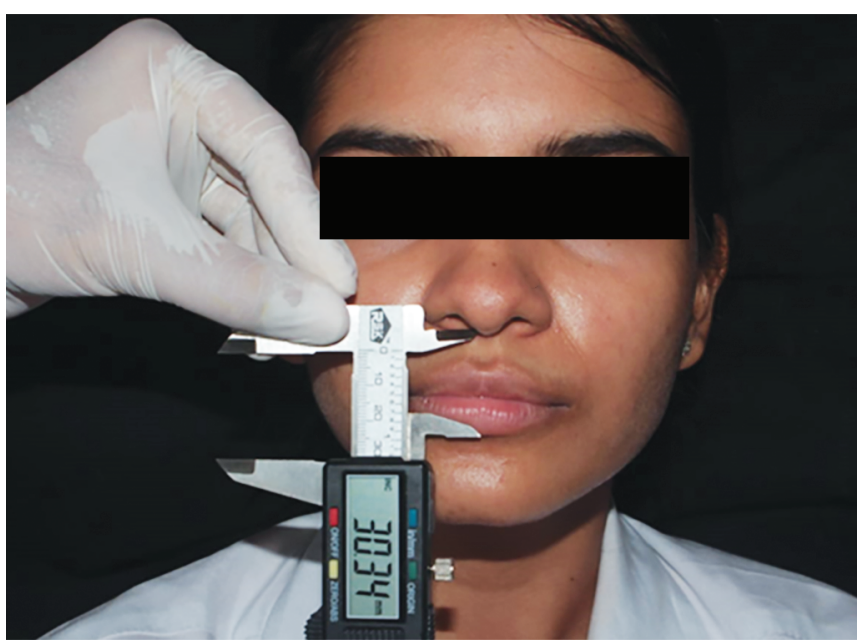

Fig. 3: Measurement of inf. border of the nasal septum to vermilion border of lower lip along the midline

- Subjects with incompetency of lips were not selected.

The ICD (distance between medial and lateral canthus of the eyes) and subnasale (base of the nose) to the lower border of lower lip distances were measured by the digital caliper when the patient closed in centric occlusion. One of the pointers was positioned at the medial canthus of the eye and the other pointer adjusted for its location at the lateral canthus of the eye. Intercanthal distance was now recorded for both left and right sides (Fig. 2). The digital caliper was then placed with its pointers between the lower border of the lower lip, and the subnasale when the maxillary jaw was in an occluded position. The distance was measured at the midline of the face (Fig. 3).

In this manner, measurements were made between the endocanthus and the exocanthus and between subnasale and labiale inferius in all the samples data obtained were tabulated for further analysis.

\section{Results}

Out of the 200 subjects, 83 were male (41.5\%) and 117 were female (58.5\%) subjects. The coincidence of occlusal vertical dimension 
with intercanthal width among the male and female categories was 76 for males and 107 for males.

Vertical dimension was equal to intercanthal width among the male $91.5 \%$ of the time and female $91.45 \%$ of the time and the degree of variation among genders was statistically insignificant.

Frequency distribution of intercanthal width was recorded in a statistically tabulated form and statistical analysis showed the mode value of the intercanthal width as $3.25-3.35 \mathrm{~cm}$ (Figs 4 and 5).

The average intercanthal width of the male subject was 3.2 $\mathrm{cm}$ and for females, it was $3.3 \mathrm{~cm}$. Female subjects showed greater intercanthal width than the male subjects.

Thus, the data collected from the study showed that the vertical dimension between the subnasale to the lower border of the lower lip was coinciding with intercanthal width $>90 \%$ of the time. The most frequently occurring intercanthus width was estimated by using the mode value and it is fell between $3.26 \mathrm{~cm}$ and $3.35 \mathrm{~cm}$. The average intercanthal width in males was $3.2 \mathrm{~cm}$ and in females were $3.3 \mathrm{~cm}$. The intercanthal width has thus proved to be a useful and reliable guide in assessing the vertical dimension of occlusion in prosthodontics.

\section{Discussion}

One of the most vital steps in complete denture fabrication is the accurate determination of occlusal vertical dimension. Regrettably, there is not a single precise method to record the same. ${ }^{2,8}$ Though researchers have challenged the theory of a constant vertical dimension at rest, it is still seen as the first step by most dentists. Tallgren observed that the position of the mandible at rest, due to its inconsistency, should not be constituted as a reliable reference position for assessment of vertical dimension during construction of complete dentures. ${ }^{12}$ Knebleman pointed out in specimens of human skulls where growth, occlusion, and development are perceivably normal, it is feasible to tie in the distance of craniofacial landmarks and use them to record measurements from the skull which can be used to help in establishing occlusal vertical dimension. ${ }^{13}$ Based on this premise, several landmarks and distances between them have been used to arrive at the vertical dimension of jaws.

Soft tissue landmarks are also commonly used to determine the vertical dimension of edentulous subjects. Willis method,
Wright's, method, and Sears methods arc based on soft tissue landmarks which help to orient the vertical dimension in artificial dentures. ${ }^{14,15}$

The Willis device was designed to aid in the measurement of the distance from the inferior border of the septum of the nose along the midline to the inferior border of the chin and the distance from the lateral canthus of the eye to the corner of the mouth when the teeth are in occlusion. Theoretically, these should be equal. However, practically the asymmetry present in faces makes the value of these average measurements with these anatomic landmarks debatable. Its accuracy is also altered by subcutaneous adipose tissue at the inferior border of the chin. In this study, the anatomical landmarks used are fairly stable and marks like canthus of the eye, lower border of the lower lip, and when compared with the other anthropometric facial measurements.

Generally, it has been the practice to compare the distance between the base of the nose and the lower border of the chin to the distances between the various facial landmarks to ascertain vertical dimension. In this study, it was possible to assess the

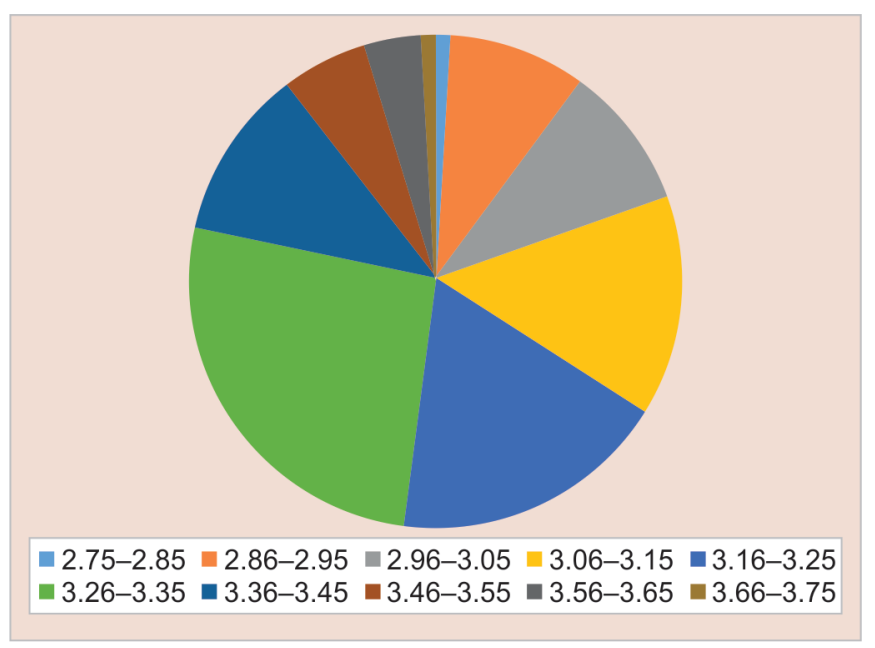

Fig. 5: Pie chart shows the distribution of the modal value of intercanthal width

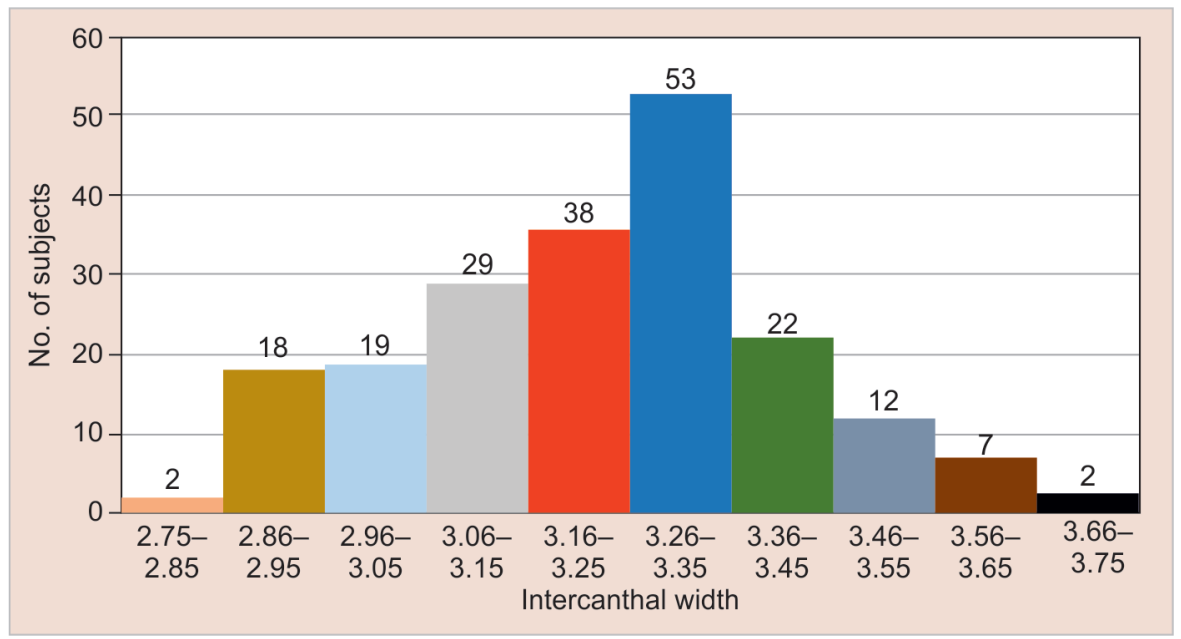

Fig. 4: Frequency distribution chart shows the intercanthal width distribution among students 
relationship between medial and lateral canthus of the eye distance and to determine the usefulness of this measurement in predicting the base of the nose-inferior border of lower lip distance for ascertaining occlusal vertical dimension in edentulous subjects.

Purely for an explanatory purpose, a pilot study was conducted and to compare this distance with a distance between maxilla and mandible to ascertain vertical dimension. It was found that the intercanthus distance closely equaled the distance between the base of the nose to the inferior border of the lower lip among 50 students. This prompted to conduct of a study among young adults to verify this hypothesis.

This study was done on young individuals where the effect of aging such as Wrinkles, folds radiating from the outer canthus of the eye are not noticed. This fact should be taken into consideration while advocating this method in very elderly subjects where such chronological changes in the skin around the eye are likely to occur. $^{16}$

The results of the study show that a significantly high percentage of both males and females showed very close coincidence to the distance between medial and lateral canthus of the eye to the base of the nose to lower border of the lower lip when mandible was in the occlusal vertical dimension. Since $91.5 \%$ accuracy was observed in both sexes it is suggested that intercanthus distance can be used as a guide to developing active vertical dimension in edentulous subjects. This can be done by adjusting the height of the occlusal rims and measuring the distance between the base of the nose to the inferior border of the lower lip till it equals the intercanthus distance. This method should be later verified by clinical judgment as well as speech test suggested by Silverman. ${ }^{15}$

The limitations encountered in the study were that samples taken were dentate subjects and of similar racial background. Thus, further studies are mandated to include different ethnic backgrounds and age-groups.

\section{Conclusion}

Within the limitations of the study, the distance between the outer and inner canthus can be a reliable landmark and can be used for anthropometric measurements to record vertical dimension in edentulous patients requiring complete dentures along with other existing methods.

\section{References}

1. de Almeida Rde C, da Rosa WL, Boscato N. The effect of occlusal splint pretreatment on mandibular movements and vertical dimension of occlusion in long-term complete denture wearers. Int J Prosthod 2016;29(3):287-289.

2. Shanahan TE. Physiologic vertical dimension and centric relation. J Prosth Dent 2004;91(3):206-209. DOI: 10.1016/j.prosdent.2003.09.002.

3. Sheppard IM,Sheppard SM. Vertical dimension measurements.JProsth Dentis 2006;95(3):175-180. DOI: 10.1016/j.prosdent.2006.01.004.

4. Strajnić $L$, Stanišić-Sinobad D, Marković $D$, et al. Cephalometric indicators of the vertical dimension of occlusion. Collegium Antropolog 2008;32(2):535-541.

5. Singh DK, Subhas S, Gupta A, et al. Facial measurements: A guide for vertical dimension. J Fam Med Primary Care 2020;9(4):2056. DOI: $10.4103 /$ jfmpc.jfmpc_968_19.

6. Majeed MI, Haralur SB, Khan MF, et al. An anthropometric study of cranio-facial measurements and their correlation with vertical dimension of occlusion among Saudi Arabian subpopulations. Open Access Maced J Med Sci 2018;6(4):680. DOI: 10.3889/oamjms.2018.082.

7. Kaimbo DK, Kayembe D. Orbital measurements in Zairian children. Inner canthal, outer orbital, inter-pupillary distances and proptosis. J Francais D'ophtalmologie 1994;17(8-9):496-500.

8. Turrell AJ. Clinical assessment of vertical dimension. J Prosth Dentis 2006;96(2):79-83. DOI: 10.1016/j.prosdent.2006.05.015.

9. Misch CE. Objective vs subjective methods for determining vertical dimension of occlusion. Quintessence Int 2000;31(4):280-282.

10. Yaw L, Subrata G, Ardan R. Determination of vertical dimension using intercanthal distance in adult Mongoloid race. Padjadjaran J Dent 2014;26(1):6-10. DOI: 10.24198/pjd.vol26no3.14003.

11. Oladipo GS, Esomonu E, Osogba IG. Craniofacial dimensions of jaw children and adolescents in Nigeria. Biomedic Int 2010;1:25-29.

12. Tallgren $A$. The continuing reduction of the residual alveolar ridges in complete denture wearers: a mixed-longitudinal study covering 25 years. J Prosthe Dentis 2003;89(5):427-435. DOI: 10.1016/s00223913(03)00158-6.

13. Knebleman $S$, The craniometric method for establishing occlusal vertical dimension. Craniometric Inc. 19.

14. Miftahullaila M, Primasari A. The Proportion of Proto Malayan's and Deutro Malayan's Vertical Dimension Using Willis's Method, Mc. Gee's Method and Golden Proportion Concept Among Students of Faculty of Denstistry In University of Sumatera Utara. IOSR Journal of Dental and Medical Sciences 2017;16(6):1-5.

15. Silverman MM. The speaking method in measuring vertical dimension. J Prosthe Dentis 2001;85(5):427-431. DOI: 10.1067/ mpr.2001.116139.

16. Ilankovan V. Anatomy of ageing face. Br J Oral Maxillofac Surg 2014;52(3):195-200. DOI: 10.1016/j.bjoms.2013.11.013. 